\title{
MHD Simulations of the ISM: The Importance of the Galactic Magnetic Field on the ISM "Phases"
}

\author{
Miguel de Avillez \\ Department of Mathematics, University of Évora, R. Romão Ramalho 59, 7000 \\ Evora, Portugal; Email: mavillez@galaxy.lca.uevora.pt \\ Dieter Breitschwerdt \\ Max-Planck-Institut für Extraterrestrische Physik, Giessenbachstraße, Postfach \\ 1312, 85741 Garching, Germany; Email: breitsch@mpe.mpg.de
}

September 29, 2003

\begin{abstract}
We have carried out 1.25 pc resolution MHD simulations of the ISM, on a Cartesian grid of $0 \leq(x, y) \leq 1 \mathrm{kpc}$ size in the galactic plane and $-10 \leq z \leq 10$ $\mathrm{kpc}$ into the halo, thus being able to fully trace the time-dependent evolution of the galactic fountain. The simulations show that large scale gas streams emerge, driven by SN explosions, which are responsible for the formation and destruction of shocked compressed layers. The shocked gas can have densities as high as 800 $\mathrm{cm}^{-3}$ and lifetimes up to $15 \mathrm{Myr}$. The cold gas is distributed into filaments which tend to show a preferred orientation due to the anisotropy of the flow induced by the galactic magnetic field. Ram pressure dominates the flow in the unstable branch $10^{2}<\mathrm{T} \leq 10^{3.9} \mathrm{~K}$, while for $\mathrm{T} \leq 100 \mathrm{~K}$ (stable branch) magnetic pressure takes over. Near supernovae thermal and ram pressures determine the dynamics of the flow. Up to $80 \%$ of the mass in the disk is concentrated in the thermally unstable regime $10^{2}<\mathrm{T} \leq 10^{3.9} \mathrm{~K}$ with $\sim 30 \%$ of the disk mass enclosed in the $\mathrm{T} \leq 10^{3} \mathrm{~K}$ gas. The hot gas in contrast is controlled by the thermal pressure, since magnetic field lines are swept towards the dense compressed walls.
\end{abstract}

Keywords: Magneto-hydrodynamics - Galaxy: disk - ISM: general - ISM: kinematics and dynamics - ISM: structure

\section{Introduction}

Modelling the ISM with high spatial resolution allows us to tackle a set of problems simultaneously, encompassing both large and small scales, provided that the appropriate grid size, resolution and numerical tools (e.g. adaptive mesh refinement) are used. To name just the most important ones, global modelling yields information on the formation and lifetimes of molecular clouds, how star-forming regions are influenced by large-scale flows in the ISM, and which dynamical rôle SNe and superbubbles play in triggering local and global star formation. In this paper we investigate the effects of the magnetic field on the dynamics and evolution of the cold gas in the disk, the relative importance of the field for the ISM "phases", and its influence on the support of shocked compressed layers.

(C) 2019 Kluwer Academic Publishers. Printed in the Netherlands. 


\section{Model and Simulations}

In the current work we report on kpc-scale simulations of the ISM on a cartesian grid of $0 \leq(x, y) \leq 1 \mathrm{kpc}$ size in the Galactic plane and $-10 \leq z \leq 10 \mathrm{kpc}$ into the halo, using a modified version of the 3D SN driven ISM model of Avillez (2000) coupled to a three-dimensional MHD code that uses adaptive mesh refinement (AMR) in a blockbased structure in combination with Message Passing Interface (MPI) suitable for massive parallel computations.

The modified model includes a fixed gravitational field provided by the stars in the disk, radiative cooling assuming optically thin gas in collisional ionization equilibrium, and uniform heating due to starlight. The radiative cooling function is a tabulated version of that shown in Figure 2 of Dalgarno \& McCray (1972) with an ionization fraction of 0.1 at temperatures below $10^{4} \mathrm{~K}$ and a temperature cutoff at $10 \mathrm{~K}$. Background heating due to starlight varies with $z$ as described in Wolfire et al. (1995); in the midplane, at $z=0$, it is chosen to initially balance radiative cooling at $9000 \mathrm{~K}$. With the inclusion of background heating the gas becomes thermally stable at $\mathrm{T} \leq 100 \mathrm{~K}$ and $10^{3.9}<\mathrm{T} \leq 10^{4.2} \mathrm{~K}$.

The prime sources of mass, momentum and energy are supernovae whose setup is similar to that described in Avillez (2000) with some modifications. In the present work SNe type Ia with a scale height of $325 \mathrm{pc}$ are also included. In regions with a density and temperature thresholds of $10 \mathrm{~cm}^{-3}$ and $100 \mathrm{~K}$, respectively, an initial mass function is applied to determine the number of OB stars and their masses, forming an $\mathrm{OB}$ association. In agreement with observations $60 \%$ of these stars explode within the association, while the remaining lowest mass stars occur in the field. The location of the field stars is determined kinematically by attributing to each star a random direction and a velocity. The associations are allowed to form in a layer with a scale height of $46 \mathrm{pc}$, while the field stars occur in a layer with a scale height of $90 \mathrm{pc}$. The time interval between the explosions of all OB stars is determined by their main sequence life time.

The interstellar gas is initially setup with a density stratification distribution that includes the cold, cool, warm, ionized and hot gas "phases" in the Galaxy as described by Ferrière (1998). In these simulations the Galactic SN rate has been used and the canonical energy of explosion is $10^{51} \mathrm{erg}$ for all types of SNe. At the beginning of the simulations the uniform field components along the three axes are given by $\vec{B}_{u}=\left(B_{u, 0}\left(n(z) / n_{0}\right)^{1 / 2}, 0,0\right)$, where $B_{u, 0}=3 \mu \mathrm{G}$ is the field strength and $n(z)$ is the number density of the gas as a function of distance from the Galactic midplane and $n_{0}=1 \mathrm{~cm}^{-3}$ is the average midplane density. The random field component is set to zero in the beginning of 
the simulations. This component is built up during the first millions of years of evolution as a result of turbulent motions, mainly induced by SN explosions. Self-gravity is not included in these calculations.

The computational grid has a resolution of $10 \mathrm{pc}$, except in the layer between -500 and $500 \mathrm{pc}$, where three levels of AMR are used, yielding a finest level resolution of $1.25 \mathrm{pc}$. Periodic boundary conditions are applied along the four vertical boundary faces, while outflow boundary conditions are imposed at the top $(z=10 \mathrm{kpc})$ and bottom $(z=$ $-10 \mathrm{kpc}$ ) boundaries. All the simulations were evolved for $400 \mathrm{Myr}$ corresponding to several sound crossing times along the fountain as required for the establishment of the duty disk-halo-disk cycle and the global dynamical equilibrium.

\section{Results}

\subsection{Global Evolution}

The initial evolution of the magnetized disk is similar to that seen in the HD runs (Avillez 2000, Avillez \& Breitschwerdt 2003), that is, the initial stratified distribution does not hold for long as a result of the lack of equilibrium between gravity and (thermal, kinetic and turbulent) pressure during the "switch-on phase" of SN activity. As a consequence the gas in the upper and bottom parts of the grid collapses into the midplane, leaving low density material in its place. However, in the MHD run it takes a longer time for the collapse to be completed as a result of the magnetic pressure and tension forces. As soon as the system has collapsed and enough supernovae have gone off in the disk building up the required pressure support, transport into the halo is not prevented, although the escape of the gas takes a few tens of Myr to occur. The crucial point is that a huge thermal overpressure due to combined supernova explosions can sweep the magnetic field into dense filaments and punch holes into the extended warm and ionized Hi layers. Once such pressure release valves have been set up, there is no way from keeping the hot over-pressured plasma to follow the pressure gradient into the halo. As a consequence the duty disk-halodisk cycle of the hot gas is fully established, which combined to the input of energy into the ISM by SNe, diffuse heating, energy lost by radiative cooling and magnetic pressure leads the system to evolve into a dynamical equilibrium state within some 200 Myr (Kahn 1981).

Fig. 1 shows slices of the three-dimensional data cube of the density and magnetic field distributions in the Galactic midplane. The highest density $\left(n \geq 100 \mathrm{~cm}^{-3}\right)$ gas tends to be confined into shocked compressed layers that form in regions where several large scale streams of 
Left panel: mavillez_fig1a, Right panel: mavillez_fig1b

Figure 1. Density and magnetic field distribution in the Galactic midplane after 374 Myr of disk evolution. The resolution of the finest AMR level is 1.25 pc.

convergent flow (associated with laminar flows driven by $\mathrm{SNe}$ ) occur. The compressed regions are filamentary in structure, tend to be aligned with the local field and are associated with the highest field strengths ${ }^{1}$. The formation time of these high density structures depends on how much mass is carried by the convergent flows, how strong compression is and on the rate of cooling of the compressed region. The compressed layers have on average lifetimes of 10-15 Myr. The streams that promote the formation of the shocked dense layers are also responsible for their destruction. The filamentary structures are thicker in these simulations than in similar HD simulations, because the compression of a shock wave at given Mach number must go into increase of thermal and magnetic pressure in the MHD case and only into thermal in HD.

\subsection{Field Dependence with Density}

The simulations started with a fixed value for the magnetic field varying with $z$ and having a midplane value of $3 \times 10^{-6} \mathrm{G}$. During the evolution of the system thermal and dynamical processes broaden the distribution of the field strength in such a way that after the global dynamical equilibrium has been set up the field strength in the disk spans two orders of magnitude from $10^{-7}$ to $10^{-5} \mathrm{G}$ as can be seen in Fig. 2. The figure shows a scatter plot of the field strength as function of density in the simulated disk after 400 Myr of evolution.

\footnotetext{
1 A similar result is seen in similar HD simulations. However, there is no preferable orientation for the filaments.
} 
Left panel: mavillez_fig2a, Right panel: mavillez_fig2b

Figure 2. Scatter plot of the magnetic field strength as a function of density for the $\mathrm{T} \leq 10^{3}$ (red) and $10^{4}<\mathrm{T} \leq 10^{5.5} \mathrm{~K}$ (black) regimes (left panel) and $10^{3}<\mathrm{T} \leq 10^{4}$ (black) and $\mathrm{T}>10^{5.5} \mathrm{~K}$ (red) regimes (right panel) at $400 \mathrm{Myr}$ of evolution.

Both panels in the figure show a large scatter in the field for the same density. This lack of correlation suggests that the field may not follow the "classical" relation of $B \sim \rho^{1 / 2}$. This essentially means that the equation of state is considerably softer than in the pure magnetic case and hence the effective adiabatic index $\gamma$ must be well below unity. This may be attributed to the variety of processes that affect the energy balance of the magnetized plasma, such as turbulence, heating and cooling. Specifically the equation of state becomes stiffer, if the dependence of the heating rate on density is steeper than that of the cooling rate.

The large scatter in the field seen in Fig. 2 also suggests that the field is being driven by the inertial motions, rather than it being the agent determining the motions. In the latter case the field would not be strongly distorted, and it would direct the motions predominantly along the field lines. The high field variability is also seen in the right panel of Fig. 1, which shows a highly turbulent field, that seems to be uncorrelated with the density.

\subsection{Pressure Variation with Temperature}

Left panel of Fig. 3 shows the scatter points of the thermal (black) and magnetic (red) pressures as a function of temperature, while the right panel shows the average magnetic, thermal and ram pressures as function of temperature. From the figure it is seen that all the $\mathrm{T} \leq$ $10^{2} \mathrm{~K}$ gas has $P_{B}>P_{\text {ram }} \gg P_{t h}$, demonstrating that magnetically dominated regions do exist, while the hot gas has the highest thermal 
Left panel: mavillez_fig3a, Right panel: mavillez_fig3b

Figure 3. Left panel shows a scatter plot of the magnetic (red) and thermal (black) pressures as functions of temperature at 400 Myr. Right panel shows the average magnetic (red), thermal (black) and ram (green) pressures as functions of temperature at 400 Myr. This plot is obtained by calculating the average pressure in a certain temperature bin. The red and black curves represent the average values of the pressures seen in the left panel.

pressure and the lowest magnetic pressure. The latter is consistent with the field strength being given by $B \sim \rho^{\alpha}$ with $\alpha \neq 0.5$. However, for $\mathrm{T}<10^{2}$ this relation does not hold, as $\alpha$ varies between -0.06 and 0.085 (Avillez \& Breitschwerdt 2004) indicating that the magnetic and thermal pressures are independent. A result that is consistent with the almost zero variation of magnetic pressure with temperature seen in the right panel of Fig. 3.

The figure also shows that for $10^{2}<\mathrm{T}<10^{6} \mathrm{~K}$ ram pressure dominates over magnetic and thermal pressures, that is, ram pressure determines the dynamics of the flow, and therefore, the magnetic pressure does not act as a restoring force as it was already suggested by the lack of correlation between the field strength and the density (for a detailed discussion see Passot \& Vázquez-Semadeni 2003). The alternating dominance between magnetic and ram pressures seen for $10^{6}<\mathrm{T}<3 \times 10^{6}$ $\mathrm{K}$ results from the fact that about half of the explosion energy in supernovae becomes kinetic, and therefore, ram pressure becomes also important near the energy sources.

The dynamical picture that emerges from these simulations is that thermal pressure gradients dominate mostly in the neighborhood of supernovae, which drive motions whose ram pressures are dominant over the mean thermal pressure (away from the energy sources) and the magnetic pressure. The magnetic field is dynamically important at low temperatures. The field does not act only by its pressure gradient, but it 
Left panel: mavillez_fig4a, Right panel: mavillez_fig4b

Figure 4. Averaged mass weighted (left panel) and cumulative (right panel) histograms of the density in the Galactic disk calculated between 300 and $400 \mathrm{Myr}$ using 101 snapshots with 1 Myr of interval. The black and blue solid lines represent gas in the thermally stable regimes at $\mathrm{T} \leq 10^{2} \mathrm{~K}$ and $10^{3.9}<\mathrm{T} \leq 10^{4.2} \mathrm{~K}$, the orange solid and dashed lines represent the thermally unstable gas with $10^{2}<\mathrm{T} \leq 10^{3} \mathrm{~K}$ and $10^{3}<\mathrm{T} \leq 10^{3.9} \mathrm{~K}$, respectively. The gas with $10^{4.2}<\mathrm{T} \leq 10^{5.5} \mathrm{~K}$ is represented by the green line, while the hot $\left(\mathrm{T}>10^{5.5} \mathrm{~K}\right)$ gas is represented by the red line.

acts dynamically also by magnetic tension forces, $1 / 4 \pi(\vec{B} \nabla) \vec{B}$. Whereas magnetic pressure is isotropic, magnetic tension is always along the lines of force. Equilibrium only exists in force-free configurations for which $\nabla \times \vec{B}=0$. However, if there is a thermal pressure then it is practically impossible to balance the magnetic stresses and most of the configurations will be unstable. In other words, in a dynamical system like the ISM, which includes systematic gas motions, $\vec{j} \times \vec{B}$-forces are inevitable, and hence the magnetic field can be dominant. Thus, regions with a high field strength such as the cold clouds should be magnetically controlled.

\subsection{Distribution of the ISM Mass}

Up to $80 \%$ of the ISM mass in the simulated disk is concentrated in the unstable regime at $10^{2}<\mathrm{T} \leq 10^{3.9} \mathrm{~K}$ (orange curves in Fig. 4) where $\sim 30 \%$ of the ISM mass is concentrated in $10^{2}<\mathrm{T}<10^{3} \mathrm{~K}$ gas (dashed orange curve), while the $10^{3}<\mathrm{T} \leq 10^{3.9} \mathrm{~K}$ gas (solid orange line) encloses some $50 \%$ of the mass. The stable regimes $\mathrm{T} \leq 10^{2} \mathrm{~K}$ and $10^{3.9}<\mathrm{T} \leq 10^{4.2} \mathrm{~K}$ enclose $\sim 1 \%$ and $\sim 10 \%$ of the disk mass, respectively. Note that most of the cold gas is concentrated in the unstable branch at temperatures $\mathrm{T} \leq 10^{3} \mathrm{~K}$ and has densities up to $100 \mathrm{~cm}^{-3}$ while gas with highest density has $\mathrm{T}>10^{3} \mathrm{~K}$. The hot gas 
amounts to less than $1 \%$, while the unstable regime with $10^{4.2}<\mathrm{T} \leq$ $10^{5.5}$ encloses $\sim 10 \%$ of the total mass.

\section{Summary}

The highest resolution MHD simulation of the ISM carried out to date, discussed in this paper, shows that large scale streams driven by $\mathrm{SNe}$ are responsible for the formation and destruction of high density clouds in shocked compressed layers. The clouds formed within the shocked gas can have densities as high as $800 \mathrm{~cm}^{-3}$ and lifetimes up to $15 \mathrm{Myr}$. Ram pressure dominates most of the coolest flows (in the unstable branch $10^{2}<\mathrm{T} \leq 10^{3.9} \mathrm{~K}$ ) except at temperatures below $100 \mathrm{~K}$ (in the stable branch), where magnetic pressure takes over.

Most of the mass in the disk is concentrated in the thermally unstable regime for $\mathrm{T} \leq 10^{3.9} \mathrm{~K}$ with some $30 \%$ of the mass enclosed in gas with $\mathrm{T} \leq 10^{3} \mathrm{~K}$. This is consistent with observations and numerical simulations discussed by Heiles (2001), Gazol et al. (2001) and Kritsuk \& Norman (2002). However, these results are certainly in disagreement with classical ISM theories (McKee \& Ostriker 1977), as from our simulations up to $90 \%$ of the ISM mass is found to be in the thermally unstable regimes with $10^{2}<\mathrm{T} \leq 10^{3.9} \mathrm{~K}(\sim 80 \%)$ and $10^{4.2}<\mathrm{T} \leq 10^{5.5}$ $\mathrm{K}(\sim 10 \%)$, where it should not exist, if it were not for the importance of dynamical processes. This calls into question the standard paradigm of an ISM distributed over three phases and being in pressure equilibrium.

\section{Acknowledgements}

We acknowledge fruitful discussions with the referee, E. Vázquez-Semadeni, and M.-M. Mac Low. MAA thanks A.I. Gómez de Castro for the financial support.

\section{References}

Avillez, M.A. 2000, MNRAS, 315, 479

Avillez, M.A., Breitschwerdt, D. 2003, A\&A (in press)

Avillez, M.A., Breitschwerdt, D. 2004, A\&A (submitted)

Dalgarno, A., McCray, R.A. 1972, ARA\&A 10, 375

Ferrière, K.M. 1998, ApJ 503, 700

Gazol, A., Vázquez-Semadeni, E., Sanchez-Salcedo, J., Scalo, J. 2001, ApJ, 557, 121

Heiles, C. 2001, ApJ, 551, L105

Kahn, F.D. 1981, in: "Investigating the Universe", ed. F.D. Kahn, Reidel Dordrecht, p. 1 
Kritsuk, A., Norman, M. L. 2002, ApJ, 569, L127

McKee, C. F., Ostriker, J. P. 1977, ApJ 218, 148

Passot, T., Vázquez-Semadeni, E. 2003, A\&A, 398, 845

Wolfire, M.G., McKee, C.F., Hollenbach, D., Tielens, A.G.G.M., Bakes, E.L.O. 1995, ApJ, 443, 152 
mavillez.tex; 3/03/2019; 20:14; p.10 
This figure "mavillez_fig1a.jpg" is available in "jpg" format from: http://arxiv.org/ps/astro-ph/0310634v1 
This figure "mavillez_fig $1 \mathrm{~b} . j p g$ " is available in "jpg" format from: http://arxiv.org/ps/astro-ph/0310634v1 
This figure "mavillez_fig2a.jpg" is available in "jpg" format from: http://arxiv.org/ps/astro-ph/0310634v1 
This figure "mavillez_fig2b.jpg" is available in "jpg" format from: http://arxiv.org/ps/astro-ph/0310634v1 
This figure "mavillez_fig3a.jpg" is available in "jpg" format from: http://arxiv.org/ps/astro-ph/0310634v1 
This figure "mavillez_fig3b.jpg" is available in "jpg" format from: http://arxiv.org/ps/astro-ph/0310634v1 
This figure "mavillez_fig4a.jpg" is available in "jpg" format from: http://arxiv.org/ps/astro-ph/0310634v1 
This figure "mavillez_fig4b.jpg" is available in "jpg" format from: http://arxiv.org/ps/astro-ph/0310634v1 